\title{
Estimation of vitamin D level in low backache cases and their outcome after treatment with vitamin $D$
}

\author{
Mohit Khanna, Amit Dahiya, Abhishek Garg*, Umesh Yadav, Ajay Sheoran, Mayank Dutta, \\ Lovjot Singh, Rakesh Sharma, Amit
}

Department of Orthopaedics, PGIMS Rohtak, Haryana, India

Received: 12 December 2020

Accepted: 19 December 2020

\section{*Correspondence:}

Dr. Abhishek Garg,

E-mail: abhi.garg003@gmail.com

Copyright: ( $)$ the author(s), publisher and licensee Medip Academy. This is an open-access article distributed under the terms of the Creative Commons Attribution Non-Commercial License, which permits unrestricted non-commercial use, distribution, and reproduction in any medium, provided the original work is properly cited.

\begin{abstract}
Background: Vitamin D has a significant role to play in bone metabolism and neuromuscular function. Several researchers have indicated that vitamin D deficiency may be possibly related to chronic musculoskeletal pain including chronic low back pain (CLBP). Objectives of this present study were conducted to rule out the vitamin D deficiency in a patient can also be a cause of low backache other than various spinal disorders like PIVD, spondylolisthesis etc.

Methods: A total of 50 patients, of any age who visited the Department of Orthopedics outpatient/Emergency, with chief complaint of low backache without any low backache disease like PIVD and spondylolisthesis etc. were thoroughly interviewed and examined for any concomitant pathological disease of spine. Patient's Blood sample of about $5 \mathrm{ml}$ with syringe of $10 \mathrm{cc}$. were taken and the serum vitamin D level was assayed by "direct competitive chemiluminescence immunoassay" (CLIA).

Results: Out of 50 patients of low backache and vitamin D deficiency, 33 patients have good outcome in their pain after getting treatment in form of Vitamin D.

Conclusions: We concluded that the vitamin D plays a major role in low backache and after treatment there is significant improvement in low backache.
\end{abstract}

Keywords: Vitamin D, Chronic low backache, Activity limitation

\section{INTRODUCTION}

Vitamin D was classified as a vitamin in the early 20th century and in the second half of the 20th century as a prohormone ("conditional" vitamin). Vitamin D has been traditionally known as "anti-ricketic factor or sunshine vitamin". ${ }^{1,2}$ Vitamin D Deficiency (VDD) is prevalent in India, a finding that is unexpected in a tropical country with abundant sunshine. Despite its sunny environment, hypovitaminosis D is common in India. According to various studies published earlier there is widespread prevalence of varying degrees (50-90\%) of Vitamin D Deficiency with low dietary calcium intake in Indian population. ${ }^{3,4}$ Chronic low back pain is a leading cause of disability, often resulting in a reduced quality of life. Up to $80 \%$ of individuals who suffer from back pain are unable to identify the cause, making it difficult to treat their pain. Researchers found that patients with chronic low back pain had significantly diminished vitamin D levels when compared with healthy controls $(p<0.0001)$. Half of the patients with low back pain were vitamin D deficient. CRP and RA levels tended to be elevated in cases when compared to controls. ${ }^{5}$

Prolonged chronic vitamin D deficiency can result in osteomalacia, moreover mild vitamin D deficiency may produce a variety of musculoskeletal pains such as fibromyalgia-like pain, low back pain, and arthralgia. ${ }^{6}$ This study was carried out to estimate the level of vitamin $\mathrm{D}$ in Low backache cases and their treatment with vitamin D. 


\section{METHODS}

A total of 50 patients suffering from low backache, who fulfilled the inclusion and exclusion criterion and who reported in the outpatient Dept. of Orthopaedics of Maharishi Markandeshwar Institute of Medical Sciences and Research, Mullana, Ambala (Haryana) were included in the study. Treatment with vitamin D was given to all the patients and improvement was captured in respective follow ups. It was a prospective review for the period of 2 years i.e. July 2014 to august 2016. Written informed consent was obtained from the patients enrolled in the study and then they were followed up. Patients of either sex, any age, willing to give informed consent to undergo study, with chronic low backache, having no pathological diseases related to spine without any radiating pain, with sign and symptoms of hypocalcemia, who received therapy to prevent or treat osteoporosis and who received vitamin D therapy but showed no improvements were included in the study.

Patients not giving consent, subjects suffering from various diseases needing various surgical orthopedics interventions, patients on palliative treatments, patients having any radiating pain with neurological deficit and patients with different orthopaedic diseases like PIVD, spondylolisthesis etc were excluded from the study.

Patients were being interviewed after checking their suitability as per the inclusion and exclusion criteria irrespective of the age, sex, religion, income, occupation, socio economic status and place of residence. The study was conducted after permission of Institutional Ethical Committee, Maharishi Markandeshwar Institute of Medical Sciences and Research, Mullana, Ambala.

At the facility, vitamin D testing is carried out using Chemiluminescence immunoassay (CLIA) technology in a fully automated analyzer advia centaur using patient's blood sample of about $5 \mathrm{ml}$. CLIA is a quantitative immunoassay method used for the determination of total $25(\mathrm{OH}) \mathrm{D}$ in serum or plasma on a fully automated platform. It is a highly sensitive technology in which a specific antibody to vitamin $\mathrm{D}$ is used for coating magnetic particles (solid phase) and vitamin D is linked to an isoluminol derivative. The light signal is measured by a photomultiplier as relative light units and is inversely proportional to the concentration of 25-hydroxyvitamin D present in the samples.

\section{Statistical analysis}

Nominal data (such as gender, complications) was presented as number and percentages. Continuous data (such as age, ALP, vitamin D) was expressed as mean, standard deviation and range. At the end of the study, the data was collected and analyzed by chi square test. A p value of $<0.05$ was considered as statistically significant.
Patients suffering from Chronic low backache.

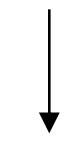

Vitamin D estimation was done for all the patients after checking inclusion and exclusion criterion.

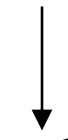

Treatment was given to all the patients.

Follow up of the patients on the same parameters.

\section{Figure 1: Workup algorithm.}

\section{RESULTS}

In our present study of 50 patients of low backache, the maximum number of patients were from the age group more than 50 years $(66 \%)$ followed by $12(24 \%)$ patients in the age group of 41 to 50 years and minimum number of patients were from the age group 31-40 compromising $10 \%$ of the total. In our study, maximum number of patients were females $[n=37(74 \%)$. Males were $26 \%$ of the total low backache patients. Maximum patients $31(62 \%)$ were from lower class, $11(22 \%)$ from middle class and $8(16 \%)$ were from high class society (socio economic status).

Maximum no. of patients i.e. (62\%) were vegetarian and $(38 \%)$ were non vegetarian. $87.09 \%$ females and $12.9 \%$ males were observed to be vegetarian. In non-vegetarian $52.7 \%$ were females and $47.3 \%$ were males. In our study, $72 \%$ patients had inadequate exposure to sunlight and $28 \%$ of patients had adequate exposure to sunlight. Out of 36 patients who had inadequate sun exposure $80.5 \%$ were females and $19.5 \%$ were males. $82 \%$ of patients had moderate pain score, $4 \%$ had severe pain score and $14 \%$ had mild pain score as per (numerical pain score) in our study. $92 \%$ patients did not complain of any radiating pain to lower limbs, only $8 \%$ of total patients complained of radiating pain. $86 \%$ of patients had limitation of daily activities due to LBA. Daily activities of $(14 \%)$ patients was not affected. $42 \%$ of our total patients had increased amount of alkaline phosphatase while $58 \%$ had no change in alkaline phosphatase value. $64 \%$ of total patients were obese and $36 \%$ were non obese.

In our study we observed that maximum no. of patients i.e. $37(74 \%)$ had their range of vitamin D in the range from 21-25. 66\% of our total patients had good improvement after treatment protocol followed by us. $24 \%$ patients had moderate improvement while $8 \%$ had mild improvement. Only $2 \%$ patients had no relief in their pain. 


\section{DISCUSSION}

Chronic low back pain is an extremely common problem in general practice, internal medicine, and spinal clinics, where the condition often is labeled idiopathic.

Vitamin D is involved in calcium haemostasis and is needed for the proper functioning of bones, muscles and nerves. It also has anti-inflammatory properties. ${ }^{2}$ Multiple factors like geographical location, sunlight exposure, malnutrition and ethnicity affect the serum levels of vitamin D. Backache is a common problem in any community, which is not given due care or may be labeled as idiopathic, out of many etiological factors a suboptimal vitamin D levels may serve as valid cause in developing countries due to poverty and malnutrition. ${ }^{7}$ No sufficient studies have been conducted to analyze the contribution of hypovitaminosis D to the etiology of chronic low back pain in populations wherein vitamin D deficiency is endemic due to insufficient sun exposure, improper diet and hormonal factors .

In our present study of 50 patients of low backache, all had vitamin d deficiency. The maximum number of patients were from the age group more than 50 years (66\%) followed by $12(24 \%)$ patients in the age group of 41 to 50 years. Minimum number of patients were from the age group 31-40 compromising $10 \%$ of the total. Our results corroborate to the results of the study done by Al Faraj et al on vitamin D deficiency and chronic low back pain which concluded that vitamin D deficiency is a major contributor to chronic low back pain in areas where vitamin D deficiency is endemic. ${ }^{8}$ Screening for vitamin D deficiency and treatment with supplements should be mandatory in this setting. In our study, $72 \%$ patients had inadequate exposure to sunlight and $68.9 \%$ of females practiced wearing burkha and becoming a cause of their vitamin d deficiency leading to low back ache. Hence our study also supports the study done by Lotfi et al on hypovitaminosis D in female patients with chronic low backache which concluded low level of vitamin d plays a significant role in causing low backache. ${ }^{9}$ Despite the sunny climate, hypovitaminosis was found prevalent among women of child bearing period, leading to chronic backache. In the present study 440 persons with low backache complaints were screened and 50 patients were found as low back pain patients, the prevalence was found out to be $8.6 \%$. Similar results were observed by many investigators including Tiwari et al and Haldiya et al who found the prevalence to be $11.1 \%$ and $6.2 \%$ respectively. ${ }^{10,11}$

In the present study, maximum.ie $33(66 \%)$ patients were more than 50 years of age followed by $12(24 \%)$ in age group of 41-40 year. The mean age for patients were 51.52 years.

Similar results were obtained by Ghai et al in their study who concluded that mean age of patients with low back pain were 43.8 years. ${ }^{12}$ Maximum patients were in the age group of 36-52 years in their study. In a similar study by Goon et al $44 \%$ of population with LBP was above 40 years. ${ }^{13}$ So, we conclude that LBP occurs in $4^{\text {th }}-5^{\text {th }}$ decades of life most commonly.

In the present study majority $37(74 \%)$ of patients were females and $13(26 \%)$ were males. Bihari et al and Banerjee et al met with similar results reporting higher prevalence in females, $17 \%$ and $34.21 \%$ respectively in their study. ${ }^{14,15}$ Higher incidence in female patients is probably explained by post-menopausal estrogen deficiency, accelerating bone resorption leading to osteoporosis and low back pain. ${ }^{16}$

In the present study majority $37(74 \%)$ of the patients were deficient in vitamin $\mathrm{D}$ in range $21-25 \mathrm{ng} / \mathrm{ml}$ followed by $11(22 \%)$ patients in range of $15-20 \mathrm{ng} / \mathrm{ml}$. Only $4 \%$ patients were in range of $26-30 \mathrm{ng} / \mathrm{ml} .41(82 \%)$ were found with moderate vitamin $\mathrm{D}$ deficiency. The findings are in conformity with a study by Siddique et al. ${ }^{17}$ who reported that $81 \%$ patients had abnormal vitamin D level $(30 \mathrm{ng} / \mathrm{ml})$ in their study.

In a study done by Rkain et al postmenopausal women, results revealed that Vitamin D deficiency $(\leq 20 \mathrm{ng} / \mathrm{mL})$ was more prevalent in women with LBP ( 79\%) compared to women without LBP $(61.4 \%) .{ }^{18} \mathrm{Al}$ faraj et al met with similar results showing $83 \%$ of patients with low back pain with hypovitaminosis D. ${ }^{8}$

In the present study all the patients (100\%) had low back pain. Similar results were observed by Siddique et al who reported $100 \%$ incidence of low back pain in his study. ${ }^{17}$ Other symptoms like paraesthesia was present in $35 \%$ of cases and fatigability in $78 \%$ of cases. In our study majority $31(62 \%)$ of patients belonged to lower class income group followed by $11(22 \%)$ from middle class income group and $8(16 \%)$ in upper class income group. Bandyopadhyay et al. ${ }^{19}$ also revealed that musculoskeletal problems were significantly more among lower income group than the workers of higher income $(8.1 \%)$. In the present study majority $17(73.9 \%)$ of the females with LBP were observing burkha. In a study by Siddique et al. ${ }^{17} 72$ (29.6\%) of patients with LBP were observing veil. In our study majority $33(66 \%)$ of patients showed good improvement after treatment with vitamin $d$ supplements and $12(24 \%)$ showed moderate improvement. Similar results were observed by $\mathrm{Al}$ faraj et al who found improvement in $95 \%$ of LBP. ${ }^{8}$ The mean level of calcium among the subjects were found to be normal i.e.10.28 $\mathrm{mg} / \mathrm{dl}$. Similar results were met by Hegazy AMS et $\mathrm{al}^{20}$ who found normal serum calcium levels $(8.9+1.2 \mathrm{mg} / \mathrm{dl})$ in LBP patients. The mean level of alkaline phosphatase was found to be normal i.e. $127.62 \mathrm{IU} / \mathrm{l}$. In contrast to our results, Hegazy et al found increased level of alkaline phosphatase $(111.2 \pm 45.4 \mathrm{U} / \mathrm{l}) .{ }^{20}$ Siddique et al found ALP was raised among $54.8 \%$ males and $47 \%$ females. ${ }^{17} 66 \%$ of the subjects were experiencing inadequate sun exposure. Similar results were seen by Hegazy et al who found $52 \%$ subjects were experiencing inadequate sun 
exposure. In our study the mean BMI of patients was 16.27 $\mathrm{Kg} / \mathrm{m}^{220}$. Hegazy et al reported majority $(71 \%)$ of patients with LBP were having BMI > $25 \mathrm{Kg} / \mathrm{m}^{2}{ }^{20}$ Similarly, Tiwari et al in their study found obese subjects to be at increased risk of developing LBP. ${ }^{10}$ Our results were further supported by the findings of Ghai et al who found that the vitamin D deficiency was more prevalent $(68 \%)$ in the overweight population $\left(25-29.99 \mathrm{Kg} / \mathrm{m}^{2}\right) .{ }^{12}$ Higher BMI was found to be associated with lower vitamin D levels. ${ }^{10}$ This has been previously reported in the literature and might be due to storage of vitamin $\mathrm{D}$ in adipose tissue. ${ }^{21}$ A higher incidence of back pain was seen in patients with high BMI's. The interplay between BMI and vitamin $\mathrm{D}$ levels and the role in causation of back pain needs to be further investigated.

Majority $43(84 \%)$ of patients experienced difficulty in performing daily activities. This finding was in conformity with the findings by Sharma et al who found that $26 \%$ subjects had to change their profession due to LBP. ${ }^{22}$ Bodhare et al reported that the most severely affected body region across one year posing considerable activity limitation was LBP. ${ }^{23}$ Monga et al in their study on impact of back muscle functions, spinal range of motion in chronic nonspecific LBP found decreasing trunk muscle endurance, muscle strength and back muscle flexibility as significant factors leading to functional disability in LBP. ${ }^{24}$

All the patients were given oral supplementation of Vitamin D for a period of 6 months in our study. After which serum vitamin D level was assessed of every patient and it was found that all patients showed improvement in vitamin D level.

At the time of start of study 41 patients complained moderate pain, 7 patients complained mild pain and 2 patients complained severe pain. After completion of study 33 patients reported good improvement, 12 patients showed moderate improvement, 4 Patients reported mild improvement and 1 patient reported no improvement in pain.

At the time of start of study 43 patients $(86 \%)$ had limitation of activities of daily living and 7 patients (14\%) had no limitation of activities of daily living. After completion of study 49 patients (98\%) reported good improvement in activities of daily living and 1 patient $(2 \%)$ reported no improvement in activity of daily living. Thus, in this prospective study of 50 patients suffering with low back pain, all patients had low vitamin d level which was improved after treatment with vitamin $d$ for a period of 6 months. After which all patients reported improvement in pain and their activities of daily living.

\section{CONCLUSION}

Treatment of low Backache has always been a question for the doctors due to its vast differential diagnosis. Treatment of low backache not only includes treating it symptomatically, but also treating the underlying pathology so that the patient gets satisfactory and lasting relief. As low back pain is multi-factorial in origin and the factors are interdependent, isolating a single pathologic cause for low back pain is difficult. Majority of patients with low back pain have vitamin D deficiency. The observations in our study revealed a high prevalence of vitamin $\mathrm{D}$ deficiency in patients presenting with chronic low back pain and, furthermore, a remarkable clinical and biochemical response is observed with oral vitamin D replacement therapy.

\section{Funding: No funding sources \\ Conflict of interest: None declared}

Ethical approval: The study was approved by the institutional ethics committee

\section{REFERENCES}

1. Holick MF. 2007 Vitamin D deficiency. N Engl J Med. 357;266-81.

2. DeLuca HF. Overview of general physiologic features and functions of vitamin D. Am J Clin Nutr. 2004;80:1689S-6S.

3. Sachan A. Gupta R, Das V, Agarwal A, Awasthi PK and Bhatia V. High prevalence of vitamin D deficiency among pregnant women and their newborns in northern India. Am $\mathrm{J}$ Clin Nutr. 2005;81;1060-4.

4. Harinarayan CV, Joshi SR. Vitamin D status in India-Its implications and remedial measures. J Assoc Physicians India, 2009;57:40-8.

5. Lodh M, Goswami B, Mahajan RD, Sen D, Jajodia N, Roy A. Assessment of vitamin D status in patients of chronic low back pain of unknown etiology. Ind J Clinic Biochem. 2015;30(2):174-9.

6. de La Jara GD, Pecoud A, Favrat B. Female asylum seekers with musculoskeletal pain: the importance of diagnosis and treatment of hypovitaminosis D. Bio Med Cent Fam Pract. 2006;7(1):4.

7. Rodrigo MD. Peak bone mass measured by phalangeal BMD and its association with nutritional status, socio economics status and physical activity: a community based cross sectional study in Galle district, Sri Lanka. 2007.

8. Al Faraj S, Al Mutairi K. Vitamin D deficiency and chronic low back pain in Saudi Arabia. Spine (Phila Pa 1976). 2003;28(2):177-9.

9. Lotfi A, Abdel-Nasser AM, Hamdy A, Omran AA, El-Rehany MA. Hypovitaminosis D in female patients with chronic low back pain. Clin Rheumatol. 2007;26(11):1895-901.

10. Tiwari RR, Mrinalini CP, Sanjay PZ . Low back pain among textile workers. Ind J Occupat Environment Medic. 2003;7(1):27-9.

11. Haldiya KR, Mathur ML, Mathur NC, Mathur A. Epidemiology of musculoskeletal conditions in India. Annual Report. 2009-2010.

12. Babita Ghai MD, Dipika Bansal MD, Raju Kanukula M. High prevalence of hypovitaminosis D in Indian 
chronic low back patients. Pain Physic. 2015;18(E853):E853-62.

13. Goon M, Ghoshal S, Chandrasekaran B, Sharma C. Prevalence of Low back pain in long distance truck drivers of mountainous terrain. Advanc Occupat Soc Organizat Ergonom. 2010:3(4):S1-4.

14. Bihari V, Keasavachandran C, Pangtey BS, Srivastva AK, Mathur N. Musculoskeletal pain and its associated risk factors in residents of National Capital Region. Ind J Occupat Environmen Med. 2011;15(2):59-63.

15. Banerjee A, Jadhav SL, Bhawalkar JS. Limitations of activities in patients with musculoskeletal disorders. Ann Med Heal Sci Res. 2012;2(1):15-9.

16. Cui Z, Schoenfeld MJ, Bush EN, Chen Y, Burge R. Characteristics of hip fracture patients with and without muscle atrophy/weakness: predictors of negative economic outcomes. $\mathrm{J}$ Med Econo. 2015;18(1):1-1.

17. Siddque SA, Malik YM. Frequency of Vitamin D Deficiency in Patients of Low Backache. Ann Pak Inst Med Sci. 2011;7(4): 208-12.

18. Rkain H, Bouaddi I, Ibrahimi A, Lakhdar T, Abouqal $\mathrm{R}$, Allali $\mathrm{F}$ et al. Relationship between vitamin D deficiency and chronic low back pain in postmenopausal women. Curr Rheumatol Rev. 2013;9(1):63-7.

19. Bandhopadhyay A, Dev S, Gangopadhyay S. A study on prevalence of musculoskeletal disorders among the coalminers of eastern coalfields of India. Int $\mathbf{J}$ Occupat Safe Heal. 2012;2(2):34-7.

20. Hegazy AM, Salama BM, Elgaml AM, Alzyat AR. Association of low back pain with vitamin D deficiency and other common risk factors: A hospital based case-control study. Europ J Prevent Medic. 2015;3(1):1-5.

21. Harinarayan CV, Ramalakshmi T, Prasad UV, Sudhakar D. Vitamin D status in andhra pradesh: A population based study. Indian $\mathrm{J}$ Med Res. 2008;127:211-8.

22. Sharma SC, Singh R, Sharma AK, Mittal R. Incidence of low back pain in work age adults in rural North India. Ind J Medic Sci. 2003;57:145-7.

23. Bodhare T, Valsangar S, Bele S. An epidemiological study of work related musculoskeletal disorders among construction workers in Karimnagar, Andhra Pradesh. Ind J Commun Med. 2011;36(4):98-9.

24. Monga A, Singh S. Impact of back muscle functions, spinal range of motion and fear avoidance beliefs on disability in chronic nonspecific low back pain. Hum Biol Rev. 2012;2(1)46-55.

Cite this article as: Khanna M, Dahiya A, Garg A, Yadav U, Sheoran A, Dutta M, et al. Estimation of vitamin D level in low backache cases and their outcome after treatment with vitamin D. Int J Res Orthop 2020;7:120-4. 